\title{
Displaying spatial epistemologies on web GIS: using visual materials from the Chinese local gazetteers as an example
}

\author{
Nung-yao Lin, Shih-pei Chen, Sean Wang, Calvin Yeh \\ Max Planck Institute for the History of Science
}

\begin{abstract}
In this paper, we introduce a web GIS platform created expressly for exploring and researching a set of 63,497 historical maps and illustrations extracted from 4,000 titles of Chinese local gazetteers. We layer these images with a published, geo-referenced collection of Land Survey Maps of China (1903-1948), which includes the earliest largescaled maps of major cities and regions in China that are produced with modern cartographic techniques. By bringing together historical illustrations depicting spatial configurations of localities and the earliest modern cartographic maps, researchers of Chinese history can study the different spatial epistemologies represented in both collections. We report our workflow for creating this web GIS platform, starting from identifying and extracting visual materials from local gazetteers, tagging them with keywords and categories to facilitate content search, to georeferencing them based on their source locations. We also experimented with neural networks to train a tagger with positive results. Finally, we display them in the web GIS platform with two modes, Images in Map (IIM) and Maps in Map (MIM), and with content- and location-based filtering. These features together enable researchers easy and quick exploration and comparison of these two large sets of geospatial and visual materials of China.
\end{abstract}

\section{Introduction}

It is increasingly common for text-based projects in digital humanities to incorporate GIS and other geovisualization techniques for data exploration and search-result displays. Conversely, imagebased projects from fields such as digital art history often require text-based finding aids (such as metadata and keywords) to facilitate data discovery. Working at the intersection between spatial humanities and geohumanities, we believe that techniques associated with historical GIS could well integrate these two approaches for data discovery and exploration. In this paper, we introduce a web GIS platform created expressly for exploring and researching - based on content and source locations - a set of 63,497 historical figures and illustrations. These figures and illustrations are extracted from a larger set of four million scanned pages from 4,000 titles of Chinese local gazetteers (difangzhi). ${ }^{1}$ Local gazetteer is a genre of Chinese local history produced between the 8th and the 19th centuries. It recorded local knowledge about places and its geographical, temporal, and jurisdictional coverages as a genre are pervasive across historical China at all scales. To date, a significant portion of extant local gazetteers has been digitized as scanned pages and have become searchable full texts. Elsewhere, we have built a set of digital tools to work with these full texts and to display search results on an interactive geovisualization. ${ }^{2}$

While primarily a textual genre, the Chinese local gazetteers also contain a number of figures and illustrations, such as maps, plants, ritual objects and so on. However, there has been little scholarly effort to work with these visual materials due to a lack of systematic tools. ${ }^{3}$ Since 2017, we have 
begun identifying such visual materials from the scanned pages of local gazetteers. This collection of extracted visual materials from local gazetteers is unprecedented and will offer new possibilities for research in Chinese history. Moreover, since a majority of these materials (e.g., administrative maps, military maps, city layouts landscape drawings, astronomy figures ${ }^{4}$ ) depict spatial configurations of places, together they reflect how government officials and local elites in historical China thought about and described their localities and provide invaluable resources for those interested in historical geohumanities.

To provide an image-based finding aid for exploring this set of visual materials in local gazetteers alongside existing text-based tools, we devised a strategy that harnesses techniques and interests from both spatial humanities and geohumanities at two separate levels and that takes advantage of Chinese local gazetteers' inherent geographical character. To be specific, this collection of visual materials is distinctive from other image collections in two ways. First, each visual material is associated with a coordinate (i.e., it is extracted from a title of local gazetteer that was compiled for a specific locality in historical China), which means that we can roughly georeference it. Second, many materials in this collection are what could be considered maps of some kind, but they largely reflect different spatial perspectives from later Chinese maps made with Western cartographic principles and techniques. To elaborate, they are hand-drawn and subjective, even for those intended to be for official purposes. For instance, administrative maps were not scaled according to distance but by relative positions of natural landscapes, which often highlight certain geographical entities but eliminate unimportant ones. Therefore, by combining image-based finding aids with georeferenced visual materials via a web GIS platform, combined with base layers of later Chinese maps, we open up new ways of studying historical localities by making explicit the tensions between spatial epistemologies across time and space.

In this paper, we summarize our workflow for creating this web GIS platform, from identifying and extracting visual materials from local gazetteers, tagging them with keywords and categories to facilitate content search, then finally georeferencing them on a web GIS platform for exploration based on both contents and coordinates. We also experimented with using neural networks to train image tagging with positive results, which led us to propose an image categorization workflow combining manual tagging and machine learning that is potentially applicable to other large image collections. In our web GIS platform, aside from content- and location-based filtering, we also created two modes, Images in Map (IIM) and Maps in Map (MIM), to display the local gazetteers images on base layers for making apparent the tension between these spatial epistemologies across time and space, which would then enable comparisons between the historical and modern spatial epistemologies.

\section{Literature}

Digitization of cultural heritage has produced large quantities of digital images from scanning or photographical artefacts. Therefore, resource owners often create web interfaces for researchers and the general public to explore such image collections. Digital images, unlike digital texts, cannot be easily searched by their contents. Therefore, major finding aids for large image collections are usually based on metadata. ${ }^{5}$ The same situation also occurs in GIS projects. ${ }^{6}$ Browsing large image collections can be realized using categorical metadata fields, such as sources, temporal periods, places or geographical regions, or thematic categorization schemes designed by 
owners or researchers. Keyword search on metadata is another important way to find images relevant to users' interests. However, creating metadata for each image to facilitate search can be very costly, and normally only large institutions can afford such investment. And although space is an important dimension for organizing knowledge and would be useful as a metadata field, unfortunately not every image collection possesses strong geospatial attributes. Therefore, displaying images according to meaningful geolocations is not always achievable.

Coming from another direction, historians who work with digitized sources have long sought to relate them to space and time, and historical GIS have provided many techniques on how to integrate spatial concepts in historical studies. Nonetheless, many scholars still struggle with locating spatial descriptions in historical sources, whether textual or visual and situating them in GIS. ${ }^{7}$ For historical maps specifically, those made without modern cartographic techniques require locating their precise location by other means before any GIS modeling.

Two examples illustrate difficulties with this type of research. In the first one, Ya-Ni Huang studied the accuracy of hand-illustrated maps from the Qing dynasty (1644-1911) by representing these illustrated, imprecise maps in GIS. ${ }^{8}$ However, this process was time-consuming and resource-intensive. It required manual examination of every potential inaccuracy in the illustrated maps. As a result, Huang was able to complete GIS modeling for only two maps from the TanHsin Archives. In the second, the research team behind the Virtual Kyoto project collected a massive amount of information and investigated their locations by referencing each other in order to recover the precise locations of archival materials. ${ }^{9}$ Using georeferenced archival materials, such as current digital maps, old topographic maps, cadastral maps, aerial photos, picture maps, street photos, landscape paintings, archaeological sites data, and historical documents, the team reconstructed a 'virtual Kyoto' in a specific time and area. These two projects demonstrate that it is time-consuming and resource-intensive to represent historical maps and that this process requires humanities scholars to collaborate with GIS experts. In fact, among the 200 papers published in the last two decades on historical GIS in Taiwan, most of the efforts were spent on investigating detailed changes in spatial boundaries, which proves that it is important but yet difficult to restore historical maps that were made without modern cartography in GIS. ${ }^{10}$

In web applications, there has been also a spatial turn. ${ }^{11}$ For example, private firm Panoramio started a service in 2005 where user's photos were displayed on a web GIS platform using userspecified geolocated tags or GPS headers embedded in the photos. Google later acquired this service and, based on it, and started Google Street View. In Google Street View, user's photos were collected and combined to produce a panorama. This service is not based on geotagging. Rather, it successfully combines photos with their geolocations and provides a better user experience. Google Indoor Map and Business View are further advances from this technology and provide panoramas for indoor spaces. ${ }^{12}$

In this paper, we propose a method that combines historical visual materials within a GIS environment, where historical maps made with modern cartographic techniques are used as contextual base-maps and other visual materials are overlaid on top. This method can extend the spatial scope of research and reduce work by humans while producing results applicable in largescale web GIS and interactive query systems. 


\section{Method}

In order to provide an image-based finding aid for exploring the visual materials in local gazetteers, we first identified and extracted visual materials from four million scanned pages of Chinese local gazetteers via a semi-automatic method. We found 63,498 pages containing visual materials and invited a group of historians to work on them. We also developed corresponding digital tools with keywords-tagging and annotation functions based on their research needs. ${ }^{13}$ While the scholars only tagged images relevant to their specific research interests, we realized that a basic knowledge organization is essential to facilitate content-based search. Therefore, we organized all 63,498 images with 16 predefined tags (see Table 1). To fully describe each image's content, we allow an image to have multiple tags.

Table 1. Tags for visual materials of Chinese local gazetteers

\begin{tabular}{|c|c|c|c|}
\hline Type & Tag & Amount & Amount used for training \\
\hline \multirow[b]{7}{*}{ Spatial } & Administrative map & 20,119 & 24 \\
\hline & City layout map & 10,542 & 22 \\
\hline & Military map & 1,677 & - \\
\hline & Landscape & 14,631 & - \\
\hline & $\begin{array}{l}\text { Building or building } \\
\text { complex }\end{array}$ & 11,241 & 24 \\
\hline & Layout diagram & 926 & 24 \\
\hline & Astronomy star map & 1,082 & 22 \\
\hline \multirow[b]{9}{*}{ Non-spatial } & Ritual & 1,200 & - \\
\hline & Musical & 394 & - \\
\hline & Agricultural & 495 & 21 \\
\hline & Military & 103 & - \\
\hline & Human figure & 631 & 28 \\
\hline & Objects & 874 & - \\
\hline & Flora and fauna & 162 & 23 \\
\hline & Chart or graph & & 22 \\
\hline & Photograph & & - \\
\hline \multicolumn{2}{|l|}{ Total amount } & 63,498 & 210 \\
\hline
\end{tabular}

While results from manual tagging are satisfactory, it is not surprising that some are inconsistent. Therefore, we started to experiment with machine learning and neural networks for a more consistent tagging model, described in $\$ 3.1$. Our results not only helped to systematically identify instances of inconsistency but also led to a tagging workflow (combining manual and machine 
learning techniques) that is applicable to other large image collections. Based on this workflow, we built a web GIS finding aid that allows for both content- and location-based search (see §3.2). We also built novel features for exploration that emphasize the spatial dimension as well as the interchange between relative and absolute spatial representations of locations.

\subsection{Automatic image tagging with neural networks}

Through our image tagging process, we learned that human tagging is time-consuming and the results are likely to be inconsistent. Therefore, we explored ways to automate tagging and experimented with neural networks. Our results were surprisingly good, which led us to propose a web-based tool and workflow that would allow users to define a project-specific tagging scheme, train automatic neural network models based on a small set of manual tagging, and propagate consistent tagging to the rest of the image collection.

In 2015, researchers at the ImageNet Large Scale Visual Recognition Challenge (ILSVRC) first reported that software exceeded human ability in image recognition. ${ }^{14}$ In 2017, 29 of the 38 ILSVRC's competing teams achieved $<5 \%$ error rate, which was lower than human's. In order to increase tagging consistency, reduce human labor, and batch-process automatically, we decided to use a machine learning method - Neural Network approach. Artificial Neural Networks (NN) are computing systems inspired by the biological neural networks that constitute animal brains. ${ }^{15}$ TensorFlow is an open-source software library for machine learning applications (including NN). ${ }^{16}$ Here we use a Convolutional Neural Network (CNN) as our model. ${ }^{17} \mathrm{We}$ use TensorFlow to experiment with automatic tagging of our images. TensorFlow contains two steps: first, it uses a small set of images to train a Neural Network model, which captures the decision-making procedure behind our image tagging; second, it then uses the trained model to tag images that were not in the training set.

\subsubsection{Training images}

We manually selected high-quality training images from our manually tagged images. We chose 210 images from a total of 9 tags, about 20 images per tag (see Table 1). We also selected a few test images to verify the obtained neural network model. The 9 tags that we have chosen out of 16 tags are those with distinct features. For example, the tag "human" and the tag "agriculture" do not have much in common, thus allowing classification with high efficacy. Meanwhile, the "ritual" tag is not selected because in Chinese rituals, often there are musical instruments, dancer and animal sacrifices involved. This could lead to undesired tag assignment with our current NN. Hence, a different design of NN should be deployed for this purpose.

\subsubsection{Accuracy of validation}

The validation module provided in TensorFlow shows that our model yielded $87.1 \%$ accuracy rate of prediction. Its high rate encouraged us to apply this model to the rest of our 63,498 images for alternative tag suggestions. By comparing the tags assigned manually and the ones suggested by the model, we were easily able to find inconsistencies and proceed to more suitable tags.

\section{$\underline{\text { 3.1.3 Examples }}$}


Below we show some examples of our result. Figure 1 gives the thumbnails of 28 training images for the tag "Human figure." Ten of them are portraits and photos. The rest are illustrations of human figures of full body-length. We then used the image of a man in full body-length on the left to test our model. The highest tag suggested by our model for this image is "Human figure" with a probability of 0.955 , while the second and third highest tags are "Flora and fauna" with 0.029 probability and "Agriculture" with 0.007 . The most likely tag for this image out-scored the rest.

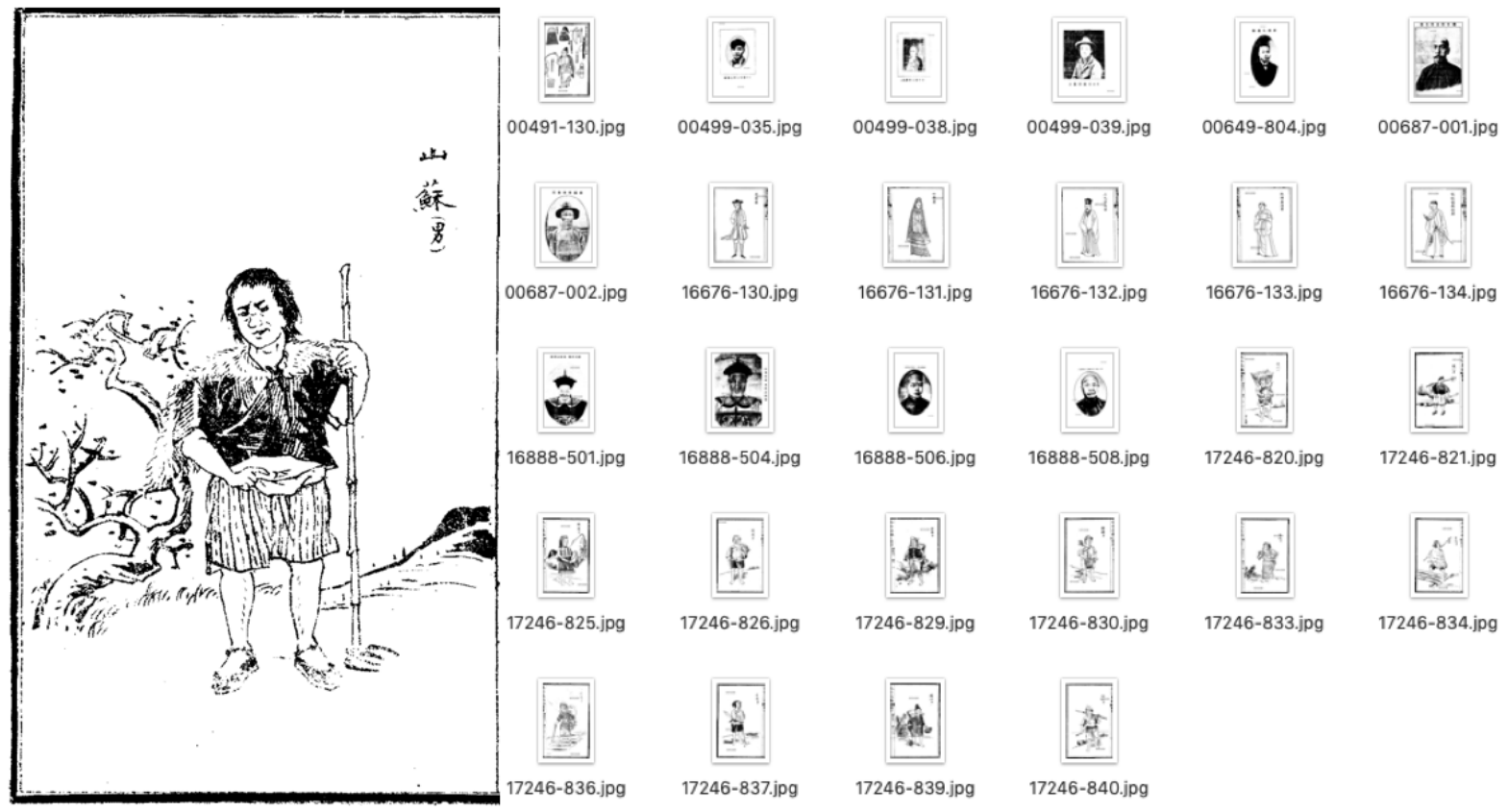

Figure 1. Training data (right) and test image (left) for tag "Human figure"

In the second test, we chose an image that in fact contains two separate images (on the left in Figure 2). One is a farmhouse and the other contains three human figures. The top three tags given by our model are: "Building or building complex" (with probability 0.271), "Human figure" (0.258) and "Agriculture" $(0.148)$. While the top two tags are with relatively low probabilities (around $0.25)$, this result correctly reflects the situation. 


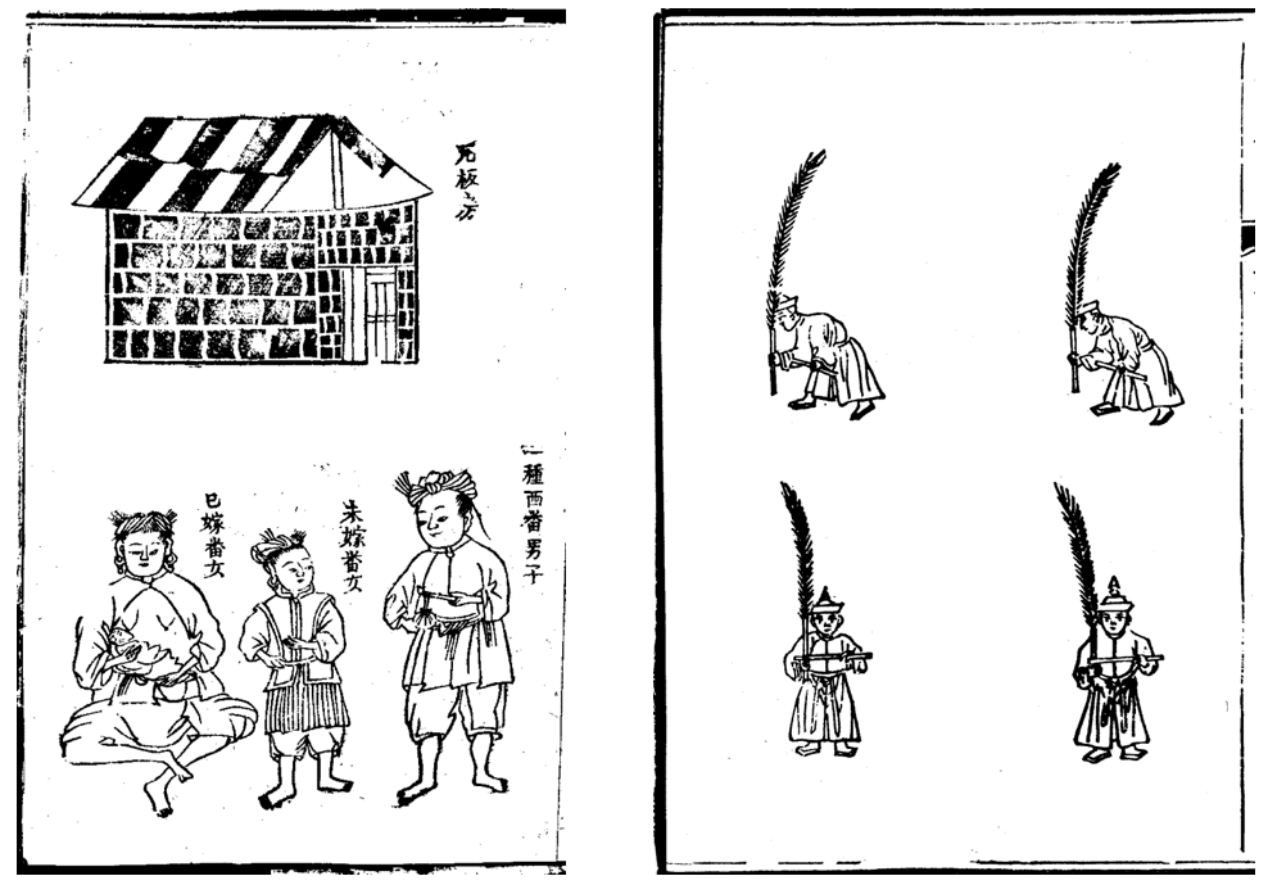

Figure 2. The 2nd (left) and 3rd (right) test images

We chose the third image from "Ritual" (on the right in Figure 2). Note that no images with this tag were included in our training dataset. This image contains four human figures demonstrating a ritual performance, where the performer holds a long ritual object that appears to be a large feather or plant. The top three tags given by our model are: "Flora and fauna" (with probability 0.864), "Human figure" (0.067), and "Agriculture" (0.029). This result very closely reflects the image content.

\subsubsection{A semi-automatic image tagging system}

Our experiment demonstrated the feasibility of combining manual tagging and neural networks to quickly propagate the model trained from a small set of manually tagged to the rest of the collection. With a friendly enough web user interface, the whole process of human tagging, training neural network models, validation and testing (of a trained model), and automatic tagging of the rest of the collection can be realized seamlessly. Such a semi-automatic tagging system might only need a small set of training images by human taggers, and thus could be an efficient basis for providing content-based search and exploration for large image collections.

\subsection{Web GIS-based finding aid}

In order to explore and study these local gazetteer images and to easily contextualize them in space, we implemented a web GIS with content- and location-based finding aids. Furthermore, our web GIS is analogous to the relationship between Google Street View and Google Maps, where users could toggle between the close and relative illustrations of space found in local gazetteers as popups, with a set of precise and scientifically produced historical maps of China as base layers. 
Overlaying the two would help examine how local communities in historical China thought about and described their lands and living spaces in relation to modern depictions of space.

We used a published collection of Land Survey Maps of China produced between the late 19th and early 20th century as the precise base-maps. From the last decades of the Qing dynasty (16441911) to the end of the Republican era (1912-1949), China's central and provincial governments and the Land Survey Department of the Japanese Army conducted geographical surveys across the country and produced maps that contain detailed topographical and administrative information. Over 4,000 of these maps were compiled and published by the Japanese publisher Kagaku Shoin between 1986 and 1998. ${ }^{18}$ They are the earliest maps of China produced with modern cartographic techniques, and they cover most of China's major cities and counties. More importantly, these maps provide a cartographic snapshot closest to an undeveloped historical China, due to the limited progress of architectural technology before China's full modernization. ${ }^{19}$

We designed this web GIS using the JavaScript library Leaflet. Users can search images from local gazetteers by tag filtering (see Table 1). The tagged images are displayed on web GIS (and clustered when appropriate) according to the geographic coordinates of their specific gazetteer title. In addition, users can also zoom to a particular region, and our tool would return images from that region. Therefore, this web GIS functions as both content- and location-based finding aids.

We provide two novel modes of displaying images to make this web GIS more useful for research purposes (see Figure 3). We call them 'Images in Map' (IIM) and 'Maps in Map' (MIM). IIM displays the thumbnails of image search results directly on a modern base-map. Through IIM, researchers can see not only the geographical distribution of the search results but also the images directly. It allows researchers to easily observe patterns and identity phenomena across geographical regions. For example, filtering for the "Human figure" tag, one can see all such images displayed on the map and can immediately observe the styles of illustrating human figures or the styles of their clothing from different regions of China.
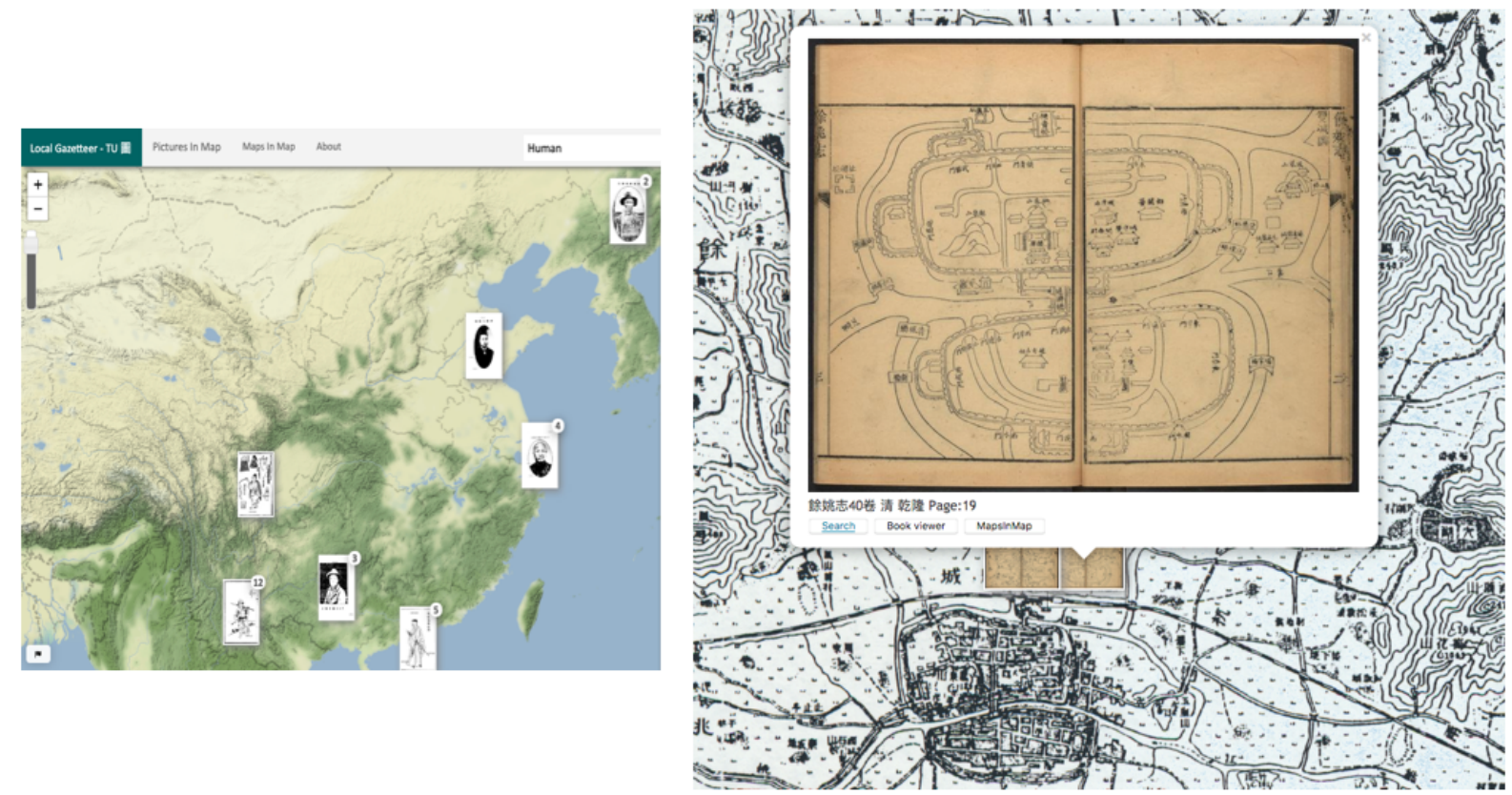
Figure 3. Examples of Images in Map (left) and Maps in Map (right) display modes

If images from search results are about spatial configurations or geographical in nature, they will be displayed as MIM. These search results would be overlaid on the land survey maps of China in order to provide more context. For example, a user can zoom to a historical city. Our interface would then show the corresponding portion of the Land Survey Maps, which are overlaid with spatial representations about this city from local gazetteers, such as city layout map and detailed buildings complex (see the right-hand side of Figure 2). Since many images from local gazetteers depict spatial configurations of places, MIM is especially useful for researchers to quickly compare the spatial depictions from the relative, illustrative historical maps given in local gazetteers and the absolute, scientific historical maps given by the Land Survey Maps. While the georeferenced Land Survey Maps represent China from the 1930s in GIS, the non-precise maps from local gazetteers actually provide more subjective details about the city, such as the relative positions of elements of the city that were important to the editors and the building facades. Therefore, the spatial visual materials from local gazetteers, despite being relative and non-precise, nonetheless provide additional details, while the Land Survey Maps, due to their consistent city layouts for military purposes, also help researchers to find the precise locations of buildings and other elements illustrated in local gazetteers.

There are also some spatial images in the local gazetteers that have even higher resolution. For example, some images depict objects for ritual ceremonies, laid out inside a temple-schoolgovernment building complex or some building interior. This level of granularity is not found in the Land Survey Maps or other scientifically produced historical maps either.

In summary, by providing both content- and location-based finding aids for historical images, displaying image thumbnails directly, and by bringing different spatial epistemologies together in one environment, we believe our web GIS interface is not just a way to digitally represent these images. Rather, it is a research tool to allow exploration of large image collections according to the spatial and bibliographical dimensions, to allow contextualization of historical images through different levels of spatial configurations, and to allow discovery of patterns. It can help scholars achieve new understandings about visual materials in local gazetteers and can inspire new research questions by treating all the images together and by putting them back into historical contexts.

\section{Displaying spatial epistemologies: an example}

In this section, we use an example to illustrate how our web GIS allows researchers to study historical China by displaying different spatial epistemologies together: namely, the modern spatial understanding of current China based on GIS and modern base maps, the georeferenced Land Survey Maps produced in the early 20th century by modern cartography, and the historical maps and spatial representations in local gazetteers.

We chose the city of Yuyao as our example. Yuyao is near Hangzhou Bay, on the south bank of the Yangzi River and is 130 kilometers south of Shanghai. The historical Yuyao was built north of the Yao River since at least the first century, and by the mid-16th century the portion on the southern bank of river was annexed. ${ }^{20}$ Today, satellite imagery shows that Yuyao has grown into a large city that encompasses land around the Yao River's various tributaries (Figure 4). However, 
it is difficult to find traces of the city's historical boundaries from the satellite imagery, except for the mountain in city center north of the river.

In our web GIS, we can zoom to Yuyao's current location and see how the surrounding region was depicted by the Land Survey Maps from the early 20th century. Figure 4 shows that in the early 20th century Yuyao remained a city divided into northern and southern parts by the Yao River, which matches the historical description from the 18th-century gazetteer. In the city map, one can find the notation of a mountain on the northern part of the city to its southwest, which provides a reference between the modern satellite imagery and the Land Survey Maps.

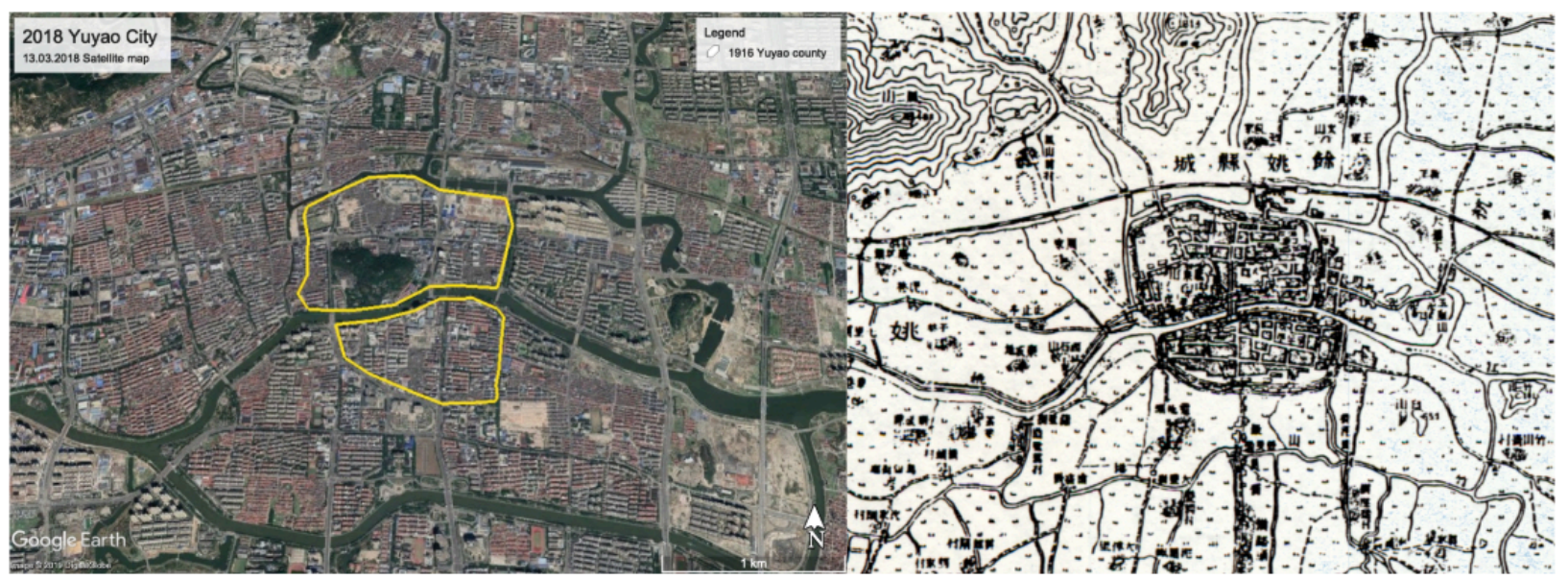

Figure 4. Birds-eye view of Yuyao city (left c.2018, right c.1916)

We can now compare the spatial images of Yuyao from local gazetteers. The 1781 Yuyao Local Gazetteer contains a sequence of hand-drawn maps (Figure 5). They are: an administrative map of the greater Yuyao region, depicting the city relative to surrounding rivers, lakes, mountains, and military bases; a city layout map depicting important elements, such as city wall, gates, roads, mountains, and buildings; layout maps of the local government building complex and the Confucius school complex; a detailed illustration of the mountain in the city; and illustrations of other surrounding mountains. Our interface can display all these spatial illustrations together with the 20th-century land survey maps. By overlaying them, researchers can switch from a modern GIS to an early modern spatial depiction of the city, then to a set of earlier historical spatial depictions about the same place. When traveling back in time, the interface could also display spatial information at different granularities. 


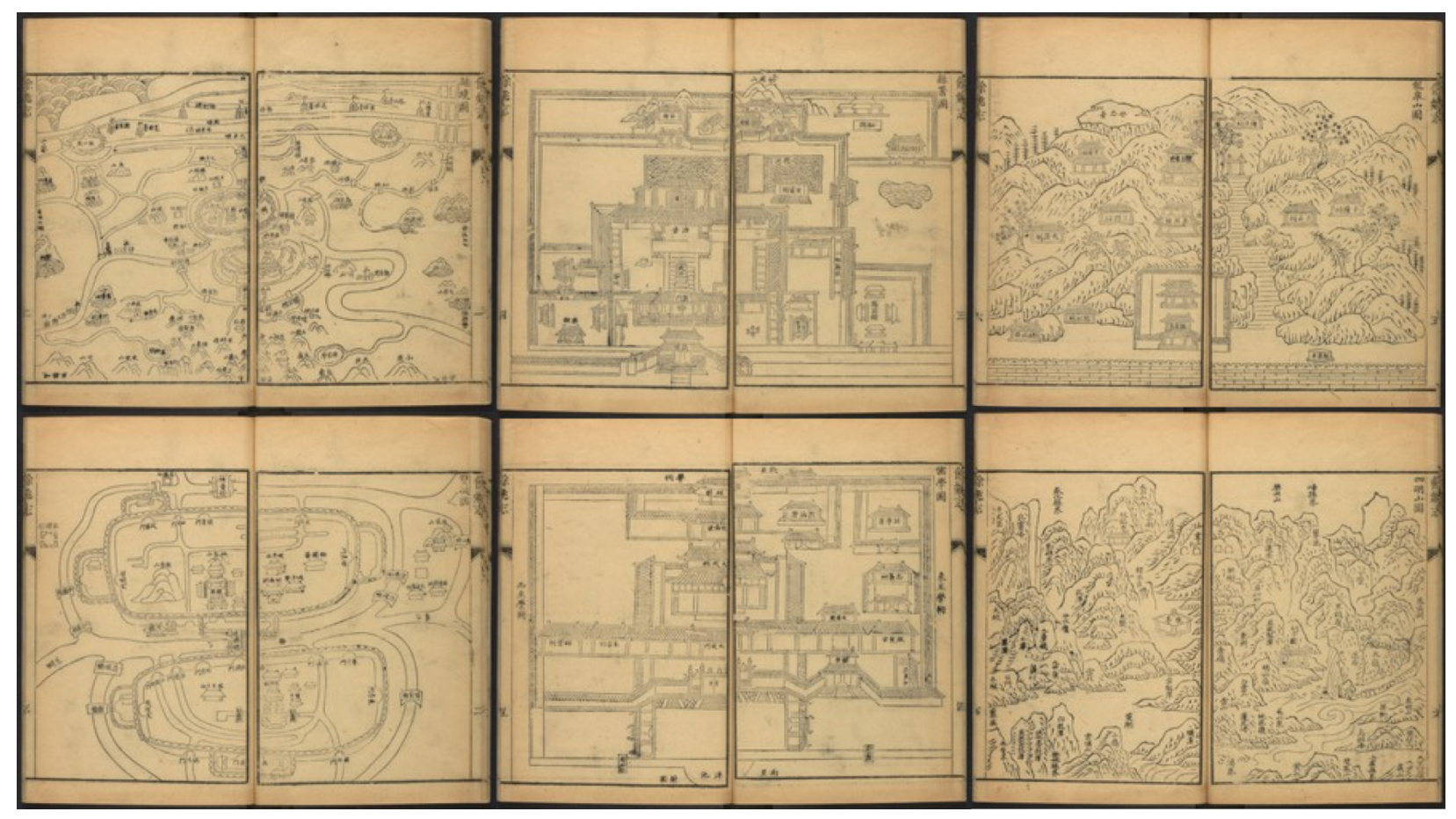

Figure 5. Spatial images in the Yuyao Local Gazetteer, 1781

From a close examination of, and comparison between, the contents of the land survey map and the city layout map from the Yuyao Local Gazetteer, we can find corresponding elements between the two. First of all, we now know more precise positions of the elements from local gazetteers and how the historical depictions are distorted from modern cartography. Second, the illustrations from local gazetteers add different layers of details to the $20^{\text {th }}$ century land survey map. Since the Land Survey Maps were produced mainly for military purposes, they omit certain details and replace them with a set of symbology. For example, the identity of buildings is as important as the routes to navigate the troops among them. Unfortunately, the symbology used in the Land Survey Maps has not been studied. The detailed illustrations in local gazetteers like administration maps, city layout maps, and buildings could actually help researchers decode the symbology used in these maps. For example, through examining the maps of Yuyao, we found that the " " symbol in the Land Survey Maps should denote post offices, “文” should mean schools, and “市” and “与” might denote Confucius and Buddhist temples respectively. Interpreting the symbols from Land Survey Maps and finding corresponding buildings from local gazetteers would further help find precise locations of buildings and advance our understanding of the city's history. There are still symbols used in Land Survey Maps that are unidentified, and further work from historians of cartography, local history, and geographers is necessary to unravel the full spectrum of these maps and images.

\section{Discussion and Conclusion}

In this paper, we made several major contributions in creating tools and methods for historians to explore a large image collection from Chinese local gazetteers. First, in order to provide contentbased finding aids for this collection, we defined sixteen tags and finished tagging for our 63,489 images by human taggers within some months. Such tags serve as categories and help to retrieve 
images for a certain type. We argue that this alternative solution is less expensive and less labor intensive compared with creating detailed metadata for each image in a large collection, an approach often adopted by libraries and museums. We further experimented with semi-automatic tagging by training a neural network using 210 images from 9 categories. Our positive results demonstrate a feasible workflow that could be applied to other large image collections to use tags as the basic content finding aid. The project owner will need to find experts to manually tag a small set of training images, but then the model can be propagated to the rest of the collection effectively and efficiently.

Based on the tags and the geographical coordinates that each image inherits from the local gazetteer to which it belongs, we built a WebGIS interface to help researchers to explore this large image collection. By displaying these historical, hand-illustrated images on modern GIS environments in the modes of Images In Map (IIM) and Maps in Map (MIM) and by allowing content- and location based filtering, our web GIS interface equips researchers with useful tools to explore this large set of images. Moreover, by bringing together spatial images given in local gazetteers and maps produced by modern cartographic techniques, researchers can study these images with different spatial epistemological backgrounds. We believe that our web GIS interface is a useful research tool that goes beyond finding aids to help researchers discover spatial patterns from this large set of local gazetteers images and to answer or raise research questions.

In the past, much of digital humanities scholarship has focused on texts, but now, by foregrounding the spatial attributes of images, we can extend the scope of research into visual materials. Machine learning can help image owners to process large number of images with relatively low cost and this makes the approach feasible for even large image collections. We also think that more machine learning methods should be explored for our images in the future, such as image recognition, image clustering (based on similarity), and search by image, especially when the cost of applying machine learning techniques decrease dramatically. By that time, we would be able to provide more ways for researchers to explore large set of images.

\footnotetext{
${ }^{1}$ This set of digitized local gazetteers is published by the Beijing Erudition Digital Technology Research Center and was made available via Staatsbibliothek zu Berlin's CrossAsia portal.

${ }^{2}$ Shih-Pei Chen et al., "Compiling a Database on Historical China from Local Records: The Local Gazetteers Project at MPIWG," in Proceedings of Digital Humanities Conference (Kraków: Jagiellonian University and Pedagogical University, 2016), https://dh2016.adho.org/abstracts/160.

${ }^{3}$ Cf. Qi Luo, "Research on the Illustrations of Chinese Local Gazetteers: Overview, Evaluation, and Potential Approach for Future Study," in Proceedings the $2^{\text {nd }}$ International Conference on Social Sciences, http://ase-scoop.org/papers/IWAHS-2016/5.Luo_IWAHS.pdf.

${ }^{4}$ Editors of Chinese local gazetteers used stars in the sky to identify the position of a place on earth. Therefore, these astronomy figures also represent a birds-eye view of local spatial configurations.

${ }^{5}$ For example, large digital image collections such as Europeana, the Library of Congress, the Vatican Library, the Kyoto University Library, and the Harvard University Library, all use some form of textbased finding aid based on metadata filtering; cf. Florian Windhager et al., "Visualization of Cultural Heritage Collections Data: State of the Art and Future Challenges," IEEE Transactions on Visualization and Computer Graphics 25, no. 6 (June 2019).

${ }^{6}$ Jennifer Marina Titus, "Peoples of Washington Historical Geographic Information System: Geocoding Culture using Archival Standards" (PhD diss., University of Southern California, 2016), 58; Jeanine Scaramozzino et al., "Map Room to Data and GIS Services: Five University Libraries Evolving to Meet
} 
Campus Needs and Changing Technologies," Journal of Map and Geography Libraries 10, no. 1 (2014): 15.

${ }^{7}$ Chung-Hsin $\mathrm{Li}$ and Ya-Wen $\mathrm{Ku}$, "Retrospect and Prospect: Application of Historical GIS to Taiwan Regional History Studies in the Last 20 Years," Taiwan Historical Research 21, no. 2 (2014): 167; May Yuan, "Temporal GIS for Historical Research," in Spatio-Temporal Narratives: Historical GIS and the Study of Global Trading Networks, ed. Ana Crespo Solana (Newcastle upon Tyne: Cambridge Scholars Publishing, 2014), 45.

${ }^{8}$ Ya-Ni Huang, "Planimetric Accuracy of Old Maps: Tan-Hsin Archives Maps" (master's thesis, National Taiwan University, 2011).

${ }^{9}$ Keiji Yano et al., "Virtual Kyoto: 4DGIS Comprising Spatial and Temporal Dimensions," Journal of Geography 117, no. 2 (2008).

${ }^{10} \mathrm{Li}$ Zongxin 李宗信 and Gu Yawen 顧雅文, “近二十年來應用歷史地理資訊系統的回顧與展望：以 臺灣區域史研究為例” [Retrospect and Prospect: Application of Historical GIS to Taiwan Regional History Studies in the Last 20 Years], 臺灣史研究 [Taiwan History Research] 21, no. 2 (2014): 171.

${ }^{11}$ Trevor M. Harris, "From PGIS to Participatory Deep Mapping and Spatial Storytelling: An Evolving Trajectory in Community Knowledge Representation in GIS," The Cartographic Journal 53, no. 4 (2016): 322.

${ }^{12}$ For more comprehensive histories of Google Street View, see Ben Campkin and Rebecca Ross, "Negotiating the City through Google Street View," in Camera Constructs: Photography, Architecture and the Modern City, eds. Andrew Higgott and Timothy Wray (London: Ashgate, 2012); Dragomir Anguelov et al., "Google Street View: Capturing the World at Street Level," Computer 43, no. 6 (June 2010).

${ }^{13}$ The working group includes four historians with their own research projects looking at visual materials from different periods and of different types; see https://www.mpiwg-berlin.mpg.de/research/projects/tutu-local-gazetteers.

${ }^{14}$ See http://image-net.org/challenges/LSVRC/.

${ }^{15}$ Warren S. McCulloch and Walter Pitts, "A Logical Calculus of the Ideas Immanent in Nervous Activity," The Bulletin of Mathematical Biophysics 5, no. 4 (1943).

${ }^{16}$ Martín Abadi et al., "Tensorflow: A System for Large-scale Machine Learning," (paper, the $12^{\text {th }}$ USENIX Symposium, 2016), https://www.usenix.org/conference/osdi16/technicalsessions/presentation/abadi.

${ }^{17}$ Yoon Kim, "Convolutional Neural Networks for Sentence Classification," Proceedings of the 2014 Conference on Empirical Methods in Natural Language Processing (2014), https://www.aclweb.org/anthology/D14-1181/.

${ }^{18}$ Chūgoku Tairiku Gomanbun No Ichi Chizu shūsei, Vol. 1-8 (Tokyo: Kagaku Shoin, 1986).

${ }^{19}$ Fuwu Luo, "Historical Development of Civil Engineering," Architecture Technology 33 (2002): 460.

${ }^{20}$ Translated from the Yuyao Local Gazetteer published in 1781: "Jiajing 36th year [1557]: Due to pirate invasion, a new city was built on the south bank of Yao river. The old and new cities are connected by Tongji Bridge, and the municipal administration expanded to cover both the south and north banks of Yao river." 